•综述・

\title{
陆地碳循环的动态非平衡假说
}

\author{
骆亦其 ${ }^{*} \quad$ 夏建阳 ${ }^{2}$ \\ 1 (Department of Biological Sciences, Northern Arizona University, Flagstaff, AZ, USA 86011) \\ 2 (华东师范大学生态与环境科学学院, 上海 200241)
}

\begin{abstract}
摘要: 生态系统维持物质与能量的动态平衡是地球系统孕育与维持生物多样性的重要基础。自工业革命以来, 人 类活动导致陆地生态系统的碳循环转变为动态非平衡, 进而使陆地生态系统的结构与功能出现许多难以预测的变 化动态。本文阐释了陆地生态系统碳循环的动态非平衡假说。该假说构建于陆地碳循环内部过程的四点基本特征 和五类外部驱动因素。基于这些内部特征与外部因素, 本文归纳了陆地生态系统碳循环动态非平衡在不同时间与 空间尺度的表达现象, 并从观测、实验与模型的角度讨论了其检测方法。陆地生态系统碳循环的动态非平衡假说 不仅有助于我们理解复杂的陆地碳循环现象, 也为预测未来陆地碳汇动态提供了新的理论框架。
\end{abstract}

关键词: 碳循环; 生态系统; 生产力; 土壤呼吸; 模型; 气候变化; 环境干扰

\section{A dynamic disequilibrium hypothesis for terrestrial carbon cycle}

\author{
Yiqi Luo ${ }^{1 *}$, Jianyang $\mathrm{Xia}^{2}$ \\ 1 Department of Biological Sciences, Northern Arizona University, Flagstaff, AZ, USA 86011 \\ 2 School of Ecological and Environmental Sciences, East China Normal University, Shanghai 200241, China
}

\begin{abstract}
The dynamic equilibrium of mass and energy movement in ecosystems is an important basis for the Earth system to nurture and maintain biodiversity. Since the Industrial Revolution, human activities have caused the carbon exchange between terrestrial ecosystems and the atmosphere to be at dynamic disequilibrium. This paper examines a dynamic disequilibrium hypothesis for the carbon cycle of terrestrial ecosystems. The hypothesis suggests that the dynamic disequilibrium is caused by interactions of four basic properties of internal processes of the terrestrial carbon cycle with five types of external drivers. Based on these internal properties and external drivers, this paper summarizes the expression phenomena of the dynamic disequilibrium of terrestrial carbon cycle at different time and space scales, and discusses its detection methods from the perspective of observations, experiments and models. The dynamic disequilibrium hypothesis for terrestrial carbon cycle not only helps us understand the complex terrestrial carbon-cycle phenomenon, but also provides a new theoretical framework for predicting the future terrestrial carbon sink dynamics.
\end{abstract}

Key words: carbon cycle; ecosystem; productivity; soil respiration; model; climate change; disturbance

地球系统可以被看作是一个进行新陈代谢的 大有机体: 生物圈在夏季吸入 $\mathrm{CO}_{2}$, 冬季呼出 $\mathrm{CO}_{2}$, 导致大气中的 $\mathrm{CO}_{2}$ 浓度呈现出夏季减少、冬季增加 的季节变化。这种季节性的呼吸模式与从太空卫星 观测到的北半球植被褐、绿转化动态相吻合。在过 去的几千年中, 地球系统的这种新陈代谢基本上一 直处于动态平衡状态。即, 碳物质在不同储存库之
间的转移速率在一定的时间尺度上保持相同, 或者 没有净变化。换言之, 在动态平衡状态下, 尽管碳 循环的所有过程一直在进行, 但是碳物质的库容量 基本保持不变。

自工业革命以来, 人类活动通过土地耕种和化 石燃料燃烧将原本储存在陆地生态系统的碳以 $\mathrm{CO}_{2}$ 的形式大量地释放到大气之中。其后果之一是导致

收稿日期: 2020-06-30; 接受日期: 2020-10-24 
地球的新陈代谢由动态平衡状态转变为动态非平 衡状态。在2009-2018年间, 化石燃料燃烧与土地利 用变化每年分别向大气释放了约 91 亿与 15 亿吨碳, 其中约32亿吨被陆地生态系统吸收, 约25亿吨被海 洋生态系统吸收, 最终使大气中每年增加了约49亿 吨碳(Friedlingstein et al, 2019)。由于陆地生态系统 吸收了大约 $30 \%$ 的人类排放的 $\mathrm{CO}_{2}$, 因此陆地的碳 汇功能是应对气候变化的一种有效且自然的解决 方案。在动态非平衡状态下, 碳物质在不同库之间 的转移速率不再相同, 因此陆地碳库的容量出现了 难以预测的变化动态。探明这些不同的转移速率如 何导致碳库变化是当今全球碳循环研究的主要目 标之一。

全球陆地碳循环进入动态非平衡状态也带来 一个现实问题: 陆地的碳汇功能在未来的全球变化 情境下是否能持久存在或者保持稳定? 近年来, 许 多研究证据表明全球环境变化正在对陆地生态系 统的碳汇功能产生重要的影响。例如, 由于干旱导 致的大面积森林死亡促使大量储存于亚马逊热带 森林中的碳以 $\mathrm{CO}_{2}$ 的形式释放到大气层(Cox et al, 2004; Brando et al, 2019); 全球气候变暖导致多年 冻土地区储存的大量有机碳被加速分解并释放到 大气层(Schuur et al, 2009; Turetsky et al, 2020)。虽然 气候变暖与大气 $\mathrm{CO}_{2}$ 浓度上升对陆地生态系统碳汇 的作用长期存在, 但是其他的干扰因子在近几十年 中也愈发重要。例如, 土地利用变化对热带森林的 干扰作用比北方森林更加显著, 导致热带森林与北 方森林对全球陆地碳汇的贡献在1992-2015年间出 现此消彼长的变化趋势(Tagesson et al, 2020)。近年 来频发的大面积火灾甚至可能使北方森林由碳汇 转变为碳源(Walker et al, 2019)。另外, 诸如风灾与 虫害等干扰因子(McDowell et al, 2020)也日益对全 球陆地生态系统的碳汇功能产生了重大威胁。然而, 当前的研究大多专注于陆地碳循环过程的测量和 数值模拟, 导致我们仍然缺少一套概念体系或者理 论框架用以陆地碳汇及其稳定性方面的研究。此外, 陆地碳循环的动态非平衡状态还与其他许多理论 问题相关。例如, 植物的光合作用产物最终以有机 物的形式储存在陆地生物圈的哪个部分? 碳原子 在陆地生态系统中的驻留时间如何影响碳循环的 动态非平衡? 驱动陆地碳循环动态非平衡的关键 生态学过程是什么? 如何利用这些过程来预测将
来碳循环的动态并制定解决减缓气候变化的碳减 排方案?

围绕以上问题, Luo和Weng (2011)提出了陆地 碳循环的动态非平衡假说。该假说认为陆地碳循环 动态非平衡动态是由两个对立的驱动力相互作用 造成的。这两个对立的驱动力是陆地生态系统碳循 环的内部平衡过程与外部的驱动变量。碳循环的内 部平衡过程包括植物的光合作用, 植物、动物和微 生物的呼吸作用，植物的生物量积累与分配，植物 调落物和土壤有机质的分解等过程。陆地生态系统 的外部驱动变量包括全球环境变化与各类干扰因 子。其中, 全球环境变化主要包括不断增加的大气 $\mathrm{CO}_{2}$ 浓度、气候变暖、降水格局变化、大气氮沉降 加剧和生物入侵等。干扰因子主要包括土地利用/ 覆盖变化(例如森林砍伐、城市化、农业种植、牧场 管理和林业经营等)，自然事件(例如虫害爆发、森林 火灾和火山爆发等)和极端天气条件(例如洪水、干 旱、台风等)。生态系统的内部过程驱使陆地碳循环 向平衡态逼近，而急剧变化的外部驱动变量则不断 导致陆地碳循环偏离平衡状态。在理想条件下，当 外部驱动变量未发生趋势性变化时, 陆地碳循环将 逐渐趋于动态平衡。反之, 当外部变量产生趋势性 变化时, 陆地碳循环将趋于动态非平衡状态。本文 在Luo和Weng (2011)一文的基础上，结合近年来的 研究进展, 重新梳理了陆地碳循环的五类外部驱动 因素, 并归纳了陆地碳循环的动态非平衡假说在不 同时空尺度的表达及检测方法。

\section{陆地碳循环内部过程的四点基本特征}

陆地生态系统中的碳循环通常起始于植物的 光合作用, 即植物吸收光能并将 $\mathrm{CO}_{2}$ 与水转化为有 机物的过程。植物光合作用的产物一部分用于植物 自身的生长，另一部分通过呼吸作用释放回大气 中。一般而言, 植物器官的存活时间从几个月(例如 叶和细根)直到数百年(例如乔木茎干)。植物枯死部 分自然脱落或由于外力作用变成调落物，从而被微 生物分解。其分解产物一部分以 $\mathrm{CO}_{2}$ 的形式释放回 大气中, 另一部分则变成土壤有机质。土壤有机质 在被氧化之前可以将碳储存在土壤中数十年甚至 长达数百或数千年(Torn et al, 1997; Trumbore, 2009; Shi et al, 2020)。以上的陆地碳循环系统具有四点基 本特征(Luo \& Weng, 2011): 
第一, 植物的光合作用为陆地生态系统碳输入 的主要来源。虽然一些土壤藻类与细菌也具有光合 作用功能, 同时干旱地区与喀斯特生态系统的土壤 也能通过化学反应吸收 $\mathrm{CO}_{2}$ 形成碳酸盐, 但是在全 球尺度上这些 $\mathrm{CO}_{2}$ 输入过程的量级较小。

第二, 陆地碳库可以依据物理边界(例如植物 的根、茎、叶、调落物和土壤)或者土壤的物理化学 性质被分隔成不同储存库, 从而使得陆地碳循环被 定义为网络化的多库系统。换言之, 光合作用所固 定的碳必须先经过这些具有不同滞留时间的碳库 网络系统，然后被依次释放回大气。

第三, 碳物质在库间转移时, 供给碳的碳库称 为供体库 (donor pool), 接收碳的碳库称为受体库 (recipient pool)。碳在不同储存库之间的转移速率主 要受供体库控制。例如, 伴随着植物的自然死亡过 程, 碳物质从植物库(即供体库)向调落物库(即受体 库)转移, 其转移速率不受调落物库大小的影响但 受供体库的调控。

第四, 调落物与土壤有机质的分解过程一般遵 循一阶线性衰减方程。不过最近有研究表明, 在有 激发效应情况下土壤有机质的分解遵循高于零阶 但低于一阶衰减动力学方程(Liang et al, 2018)。

在以上四点基本特征中, 第一和第二点基本上 没有异议, 二者共同决定了陆地碳循环为一个单源 网络化的多库循环系统。尽管如此, 单源多库系统 的理论性质基本没有被深入研究。第三点特征对决 定陆地碳循环动态的变化轨迹极其重要。也正是因 为这一特征, 陆地碳循环在没有外部条件的扰动下 趋向平衡。目前已发表的调落物分解数据与土壤有 机质分解实验几乎都支持第三点特征的准确性 (Zhang et al, 2008; Yang et al, 2011; Xu et al, 2016; Cai et al, 2018)。最近, 有一种观点认为在土壤有机 质的分解过程中微生物既是受体又是供体, 并由此 推导出一套非线性的土壤微生物模型(Allison et al, 2010; Weintraub \& Schimel, 2003; Wieder et al, 2013)。基于这些模型的数学原理, 陆地碳循环将不 一定向平衡态逼近而是产生各种复杂的动态轨迹 (例如振荡, Wang et al, 2014)。然而, 目前仍没有观 测数据和实验证据支持陆地碳循环遵循如此复杂 的动态轨迹。

基于以上的四点基本特征, 陆地生态系统碳循 环的过程可以用以下数学方程式表示(Luo et al,
2003; 2017; 图1):

$$
\frac{d \boldsymbol{X}(t)}{d t}=\mathbf{B} U(t)+\mathbf{A} \xi(t) \mathbf{K} \boldsymbol{X}(t)
$$

其中 $\boldsymbol{X}(t)$ 代表生态系统各个碳库大小的向量, $U(t)$ 是 光合作用的输入或生态系统净初级生产力, $\mathbf{B}$ 为光 合产物在各个碳库的分配系数向量, $\mathbf{K}$ 和 $\mathbf{A}$ 分别代 表各个碳库的分解速率矩阵与碳库间的转移矩阵, $\xi(t)$ 是环境标量。方程1可以描述大多数观察到的碳 循环过程, 例如调落物和土壤有机碳的分解过程 (Silver \& Miya, 2001; Zhang et al, 2008; Xu et al, 2016; Cai et al, 2018)。该方程是大多数生态系统碳 循环模型的基础(Parton et al, 1987; Lawrence et al, 2020), 比较完整地刻画了碳循环的四点基本特征。

相比之下，在种群动态模型中，捕食者与猎物 的种群动态受到供体与受体的共同控制，导致捕食 系统的种群动态较为复杂(Holling, 1959)。在公式(1) 中, 陆地碳循环的动态主要由供体库主导, 因此在 $U(t)$ 不发生剧烈变化且没有其他外部扰动的情况下, 陆地碳循环的动态长期趋向平衡。陆地碳循环的平 衡机制最初由Odum (1969)描述，可以通过数学分 析和观测数据进行验证。在数学上, 公式(1)描述了 一个满足指数型收敛于平衡态的非自治系统 (Rasmussen et al, 2016)。根据观测数据, 许多研究表 明植物和土壤库中的碳储量在森林的次生演替过 程和草地在干扰后的恢复过程中都逐渐趋向平衡 态演变(Johnson et al, 2004; Matamala et al, 2008; Yang et al, 2011)。

\section{陆地碳循环的五类外部驱动因素}

全球环境的剧烈变化对陆地生态系统的结构 与功能将产生深远影响。我们把陆地生态系统可能 会经历的环境变化情景分为五类(图1), 简述如下:

第一类是周期性的环境变化，例如光照、温度 和其他环境因子在昼夜和季节尺度上的周期性变 化。由于光照与温度等因子调控着大多生物学过程, 因此导致陆地碳循环过程产生周期性变化节律。例 如, 光照和温度在夏季远高于冬季, 因此植物的光 合作用速率也呈现夏高冬低的季节动态。在这种周 期性变化的任何时刻, 只要光合作用的碳输入不等 于呼吸作用的碳释放，碳循环就处于非平衡状态。 然而, 如果该周期性变化的节律本身不变, 碳循环 


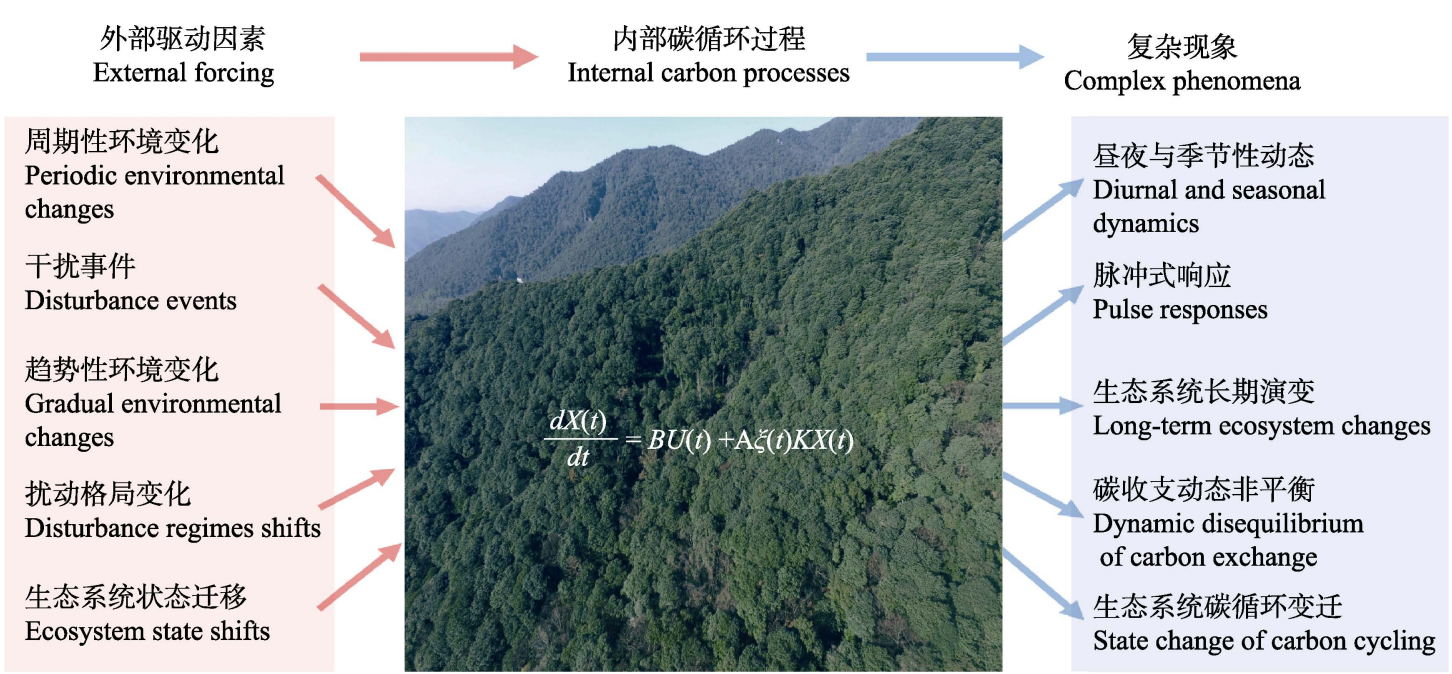

仍旧处于动态平衡, 即长期的光合作用碳输入约等 于呼吸作用碳释放。随着全球气候变暖, 地球表面 温度的周期性节律发生变化, 导致碳循环可能无法 处于动态平衡状态。例如, 近期大量的观测数据表 明陆地碳循环的年际变异越来越大(Piao et al, 2020), 表明全球碳循环系统可能由动态平衡状态正在转 变为动态非平衡状态。

第二类环境变化情景是扰动事件, 例如火烧和 极端天气与气候事件(Running, 2008; Mack et al, 2011; Reichstein et al, 2013; Walker et al, 2019)。在群 落生态学中, 此类单次扰动事件也被称为脉冲式干 扰事件(pulse perturbation), 可对生态系统的稳定性 产生重要影响(Donohue et al, 2016)。对碳循环而言, 火烧事件本身导致植物地上生物量和调落物层中 的碳被大量快速释放(Walker et al, 2019)。观测数据 表明, 随后的几年至几十年或者几百年中植物生长 和土壤碳含量会逐步恢复(Odum, 1969; Yang et al, 2011; Williams et al, 2012; Zhu et al, 2018)。如果生 态系统能恢复到扰动前的状态, 那么陆地碳循环仍 长期处于动态平衡。然而, 如果气候变化增加了扰 动事件的发生频率与强度, 可能使生态系统无法恢 复到扰动前的状态, 进而导致陆地碳循环处于动态 非平衡状态(Walker et al, 2019)。

第三类是趋势性的环境变化, 包括大气中的
$\mathrm{CO}_{2}$ 浓度逐步上升、气候变暖、降水格局的改变和 氮沉降加剧。这些趋势性的全球环境变化因子共同 导致陆地碳循环的通量、滞留时间和储量偏离了平 衡状态。例如, 大气 $\mathrm{CO}_{2}$ 浓度的上升直接刺激光合 作用, 从而增加生态系统的碳输入量(Franks et al, 2013)。全球环境变化对陆地碳循环的直接影响一般 是指碳循环过程对环境因子的直接响应(Burke et al, 2003)。但是，环境变化也对陆地碳循环产生许多间 接影响, 例如改变植物物种组成和生物多样性 (Hautier et al, 2015)。相对于碳循环过程, 植物群落 组成和物种多样性变化对全球环境变化的响应较 慢，因此可能是碳循环处于动态非平衡状态的间接 原因之一。然而, 生态学领域对群落结构如何间接 影响生态系统碳循环的理解还不是很深刻。

第四类外部环境变化情景是扰动格局 (disturbance regime)的变化。扰动格局是指扰动事件 长期发生在某个区域范围的概率格局(Hu et al, 2010)。自然扰动事件一般是随机过程，其格局一般 包含四个特征，即扰动频率、严重程度、持续时间 和扰动范围(Donohue et al, 2016)。对某一种扰动事 件而言, 例如风⿺ 目风或者火灾, 地球上的每个地区都 有其特定的格局(Vanderwel et al, 2013)。这些特定的 扰动格局可以通过扰动事件特征的联合概率分布 来量化(Weng et al, 2012)。但是，量化这种联合概率 
分布需要区域性的长期数据(Grace et al, 2014)。将 扰动格局的联合概率分布和碳循环模型互相结合 可以生成生态系统的碳储量概率分布(Weng et al, 2012), 其均值可定义为给定扰动格局下可实现的 生态系统碳储存容量(Luo \& Weng, 2011)。简言之, 在不考虑其他环境变化情景的条件下, 当某个地区 的扰动格局处于平稳态时, 陆地碳循环处于动态平 衡, 反之陆地碳循环则处于动态非平衡。

第五类是生态系统的状态变化。生态系统状态 (ecosystem state) 通常是由植被状态和土壤理化性质 决定, 其变化大多由气候变化和环境扰动引起 (Staver et al, 2011)。例如, 人类活动把森林或草地变 成农田直接导致生态系统状态发生变化(Houghton et al, 2012), 木本植物入侵草原系统也导致其生态 系统状态变化(Higgins \& Scheiter, 2012)。此外, 由 于生物量的积累, 火灾和气候变化的相互作用导致 草地与森林生态系统可能会在多个平衡状态之间 交错演替(Staver \& Levin, 2012; Resco de Dios, 2020)。生态系统状态变化通常会改变陆地碳循环的 许多过程, 例如植被的光合作用、调落物分解和土 壤微生物碳利用效率等。全球动态植被模型通常通 过模拟植被结构的变化, 刻画生态系统状态的改变, 从而预测陆地碳循环的相关过程(Franklin et al, 2020)。

\section{3陆地碳循环动态非平衡在不同时空尺 表达}

对陆地生态系统而言, 不论是碳循环的内部过 程, 还是外部的环境变化因子, 总是发生于不同的 时间与空间尺度。因此, 陆地碳循环的动态非平衡 状态在不同的时空尺度上呈现出多种表达现象。我 们结合陆地碳循环的四点内部特征与五类环境变 化情景, 总结了陆地碳循环动态非平衡在五种时空 尺度上的表现特点(图1)。

第一种, 生态系统的碳源汇状态在昼夜与季节 尺度上呈现出的周期性交替现象。主导这一现象的 主要原因是生态系统中的光合与呼吸作用对光照 与温度的周期性变化十分敏感。例如, 植物叶片的 $\mathrm{CO}_{2}$ 交换过程在白天表现为光合吸收，但是在夜间 表现为呼吸释放。因此, 生态系统在生长季中表现 为白天是碳汇而夜间转变为碳源。在季节尺度, 非 热带地区的生态系统大多表现为夏季是碳汇而冬
季转变为碳源。然而, 由于碳原子在不同碳库的滞 留时间不一致，因此不同碳库对环境变化的响应强 度存在差异。例如, 叶片的碳滞留时间一般为数月 到数年, 因此其碳库大小的周期性特征趋近于光照 与温度。土壤有机质的碳滞留时间一般为几十年至 数百年, 因此其周期性振幅对环境因子的响应强度 较弱。长期以来, 数学模型对光合与呼吸作用在昼 夜和季节尺度的周期性变化作出了很好的预测 (Farquhar et al, 1980)。因此，对于此类碳循环过程， 人们较少运用陆地碳循环的动态非平衡假说, 以增 加其可预测性。

第二种, 生态系统对局域尺度单次扰动事件的 脉冲式响应现象。当扰动事件(如火烧、虫害与极端 干旱等)在局域尺度发生时, 生态系统碳循环过程 一般表现出先下降后逐步恢复的脉冲式响应。这种 脉冲式的响应模式的时间跨度从数天到数年, 例如 单片森林的次生演替(Yang et al, 2011)与局域草地 的恢复(Matamala et al, 2008)。以极端天气与气候事 件为例, 虽然其发生频率与强度在全球尺度都呈现 增加趋势, 但是发生的时间与地点具有较大的随机 性(Du et al, 2018)。例如，欧洲中部在过去20年中经 历了2003、2010与2018年三次夏季热浪事件，对生 态系统生产力产生了不同的影响 (Bastos et al, 2020)。近期的一些群落生态学研究表明, 维持群落 多样性有助于缓冲生态系统生产力对此类单次扰 动事件的响应(Pires et al, 2018), 表现为更强的抵抗 力与恢复力(Isbell et al, 2015)。由于生产力对生态系 统碳循环的重要作用(公式1), 因此保护生物多样性 将有助于降低生态系统碳循环对单次扰动事件的 脉冲式响应强度。

第三种，陆地碳循环在全球尺度的长期趋势性 渐变。在大气 $\mathrm{CO}_{2}$ 浓度上升、气候变暖、降水格局 改变和大气氮沉降加剧等长期环境变化的共同影 响下, 陆地碳循环在全球尺度出现了许多长期性的 渐变现象。例如, 20世纪80年代以来, 陆地表面的植 被绿度(Zhu et al, 2016)与生长峰值(Huang et al, 2018)呈现逐渐升高的趋势。这些长期变化的主要环 境驱动因子是大气 $\mathrm{CO}_{2}$ 浓度上升(Piao et al, 2020)。 与此同时, 全球陆地的碳汇能力自 20 世纪 50 年代以 来也呈现增强的趋势(Ballantyne et al, 2012，2017; Ciais et al, 2019)。近期的研究发现，陆地碳汇的年 际变异在区域尺度主要受控于降水变化, 而在全球 
尺度主要由温度的年际变化主导(Jung et al, 2017)。 这说明陆地碳循环的动态非平衡状态在全球尺度 上渐变信号主要受到大气 $\mathrm{CO}_{2}$ 浓度上升和气候变暖 的驱动(Fernández-Martínez et al, 2019)。然而值得注 意的是, 随着全球干旱事件逐渐增多(Huang \& Xia, 2019), 土壤水分储量也逐渐在全球尺度影响陆地 碳汇的年际变异(Humphrey et al, 2018)。这些新研究 的发现预示着区域尺度的生态系统碳循环动态非 平衡对全球碳汇的长期趋势性变化将产生重要的 影响。需要注意的是, 人们对生物群落结构如何调 控陆地碳循环在全球尺度上的渐变趋势依然理解 不深。一方面, 植物与微生物群落结构响应环境变化 的时间尺度往往比生理生态学过程更长, 从而在不 同区域对碳循环存在许多未知的间接影响。另一方 面, 公式(1)中的各个关键过程都与植物与微生物群 落的组成结构密切相关, 但是仅有少数全球变化生 态学实验同时监测了生物群落结构与碳循环过程。

第四种, 区域尺度多重干扰强迫下的碳循环动 态非平衡现象。在区域尺度上, 环境扰动格局的形 成与变化往往需要几十年、数百年甚至几千年。以 位于北半球高纬度地区的阿拉斯加高寒生态系统 为例, 气候变暖尽管有利于植物生长, 但将使该地 区出现更多的冬季低温事件和夏季洪涝事件(Lader et al, 2017); 同时, 气候变暖也加速了该地区永久 冻土层的融化, 并提高了冻土突然融化事件的发生 频率(Turetsky et al, 2020)。此外，基于古气候与古木 炭的数据分析发现该地区的野火发生频率随着气 候变化正在迅速上升(Hu et al, 2010; Kelly et al, 2013)。这些多重扰动事件将促使北半球高寒苔原与 北方森林生态系统的碳循环进入动态非平衡状态, 因此使北半球高纬度地区的碳循环更加难以预测 (Grosse et al, 2011; Schuur et al, 2015)。事实上，在当 前的气候变化趋势下, 多重干扰强迫在全球不同区 域以不同的组合形式存在, 因此增加了在全球尺度 上理解和预测陆地碳循环动态非平衡状态的难度。

第五种, 陆地生态系统状态突变带来的碳循环 变迁现象。生态系统一般存在多种稳定状态 (alternative stable states; Holling,1973; Scheffer et al, 2001)。生态系统在不同稳态间的变迁往往具有突然 性, 且经常不依赖于环境变化, 近年来引起了生态 学领域的关注(Hastings et al, 2018; Morozov et al, 2020)。目前, 有关陆地生态系统状态突变的研究大
多关注植物种群与群落动态的变化, 而对碳储量等 生态系统状态量关注较少。因此, 当植物群落由一 种稳态突变到另一种稳态时，其内部的碳循环过程 将发生急剧变迁。在时间尺度上，人类活动主导的 生态系统状态突变往往发生速度较快且缺乏渐变 式的预警信号。例如, 在森林生态系统经砍伐转变 为农田生态系统的状态突变过程中, 生态系统碳循 环会迅速出现生产力下降、土壤呼吸升高及碳汇转 为碳源等变迁现象。而自然环境变化带来的生态系 统状态突变一般需要经历很长时间, 并伴随渐变式 的预警信号。对陆地碳循环的动态非平衡而言, 森 林的死亡过程既提供了植物种群与群落的状态突变 信息，也是生态系统碳循环状态变迁的重要预警信 号。例如, 由于全球森林的大面积死亡事件逐渐增多, 森林生态系统的碳滞留时间呈现出普遍下降的趋势 (Yu et al, 2019)。需要指出的是, 虽然生态系统状态 突变对陆地碳循环动态非平衡存在强烈影响, 但人 们对二者之间的关系和作用机制仍然知之甚少。

\section{陆地碳循环动态非平衡的检测与分析方}

由于陆地碳循环包含大量内部生物学过程, 因 此近年来在不同时空尺度涌现出丰富的观测与实 验手段(Xia et al, 2020)。我们将这些方法归纳为观 测、实验与模型三类, 并介绍如何运用这些研究手 段监测陆地碳循环的动态非平衡状态(图2)。

陆地碳循环动态非平衡状态的重要测量指标 是净生态系统生产力(net ecosystem productivity, $\mathrm{NEP}$ ), 通常是指生态系统在一年中的净 $\mathrm{CO}_{2}$ 交换 量。换言之, 陆地碳循环动态非平衡状态是指NEP 在年际间存在波动，且在一段时期内不等于零。在 站点尺度，基于浴度相关技术的通量观测塔是目前 监测NEP动态的主要手段(Baldocchi, 2020)。在全 球 尺度, 以FLUXCOM为代表的数据产品结合了机器 学习算法和全球联网的通量观测数据(Jung et al, 2020), 已被广泛应用于全球陆地碳循环方向的研 究。此外, 基于大气 $\mathrm{CO}_{2}$ 观测数据反演的全球陆地 $\mathrm{NEP}$ 数据也常被用于碳源与碳汇动态的监测研究 (Rödenbeck et al, 2018)。近年来兴起的卫星遥感技 术，为监测全球陆地生态系统的植被动态与生产力 变化提供了大量数据。例如, 基于卫星遥感数据估 算的雨水利用效率，可以指示生态系统功能在极端 

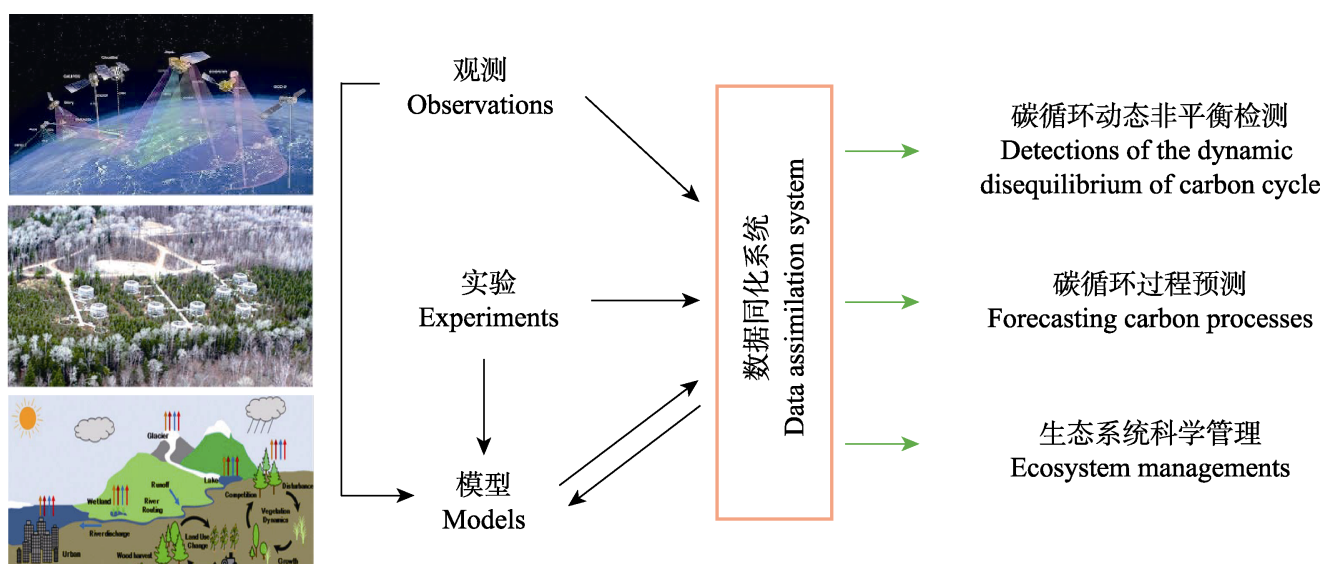

图2 陆地碳循环动态非平衡状态与相关过程的检测与分析方法

Fig. 2 Detections and analyses of the dynamic disequilibrium state and related processes for terrestrial carbon cycle

干旱年份受损情况(Du et al, 2018), 并以此定位极 端干旱事件导致陆地碳循环动态非平衡的发生区 域。此外, 长期的野外定位观测和大尺度的野外复 查工作也是监测陆地碳循环动态非平衡状态的重 要方法。

全球变化控制实验模拟了生态系统对特定环 境变化因子的响应, 可以用于检测陆地碳循环是否 在未来全球变化情景下出现动态非平衡状态。最近 的一项整合分析研究发现, 大部分全球变化因子 (例如大气 $\mathrm{CO}_{2}$ 浓度上升、气候变暖、降水增加和大 气氮沉降加剧)都加速了生态系统碳循环的过程 (Song et al, 2019)。其中，一些持续时间较长的控制 实验发现碳循环对全球变化的响应处于动态非平 衡状态。例如, 在一项位于美国哈佛森林的增温实 验中, 土壤呼吸对增温连续26年的响应动态呈现出 动态非平衡的特征(Melillo et al, 2017)。近年来, 许 多野外控制实验开始考虑极端性气候/天气事件 (Knapp et al, 2017)和火烧等干扰事件(Hu \& Wan, 2019)对生态系统碳循环的影响。这些实验为检测陆 地碳循环动态非平衡假说提供了非常丰富的数据 和研究平台。

地球系统模式(Earth System Models)是分析与 预测陆地碳循环动态非平衡状态的重要工具。地球 系统模式耦合了地球系统中的动力、物理、化学和 生物学过程, 同时模拟了陆地碳循环的外部环境变 化与内部动力学过程。事实上, 公式(1)可以描述目 前大部分地球系统模式中的陆地碳循环模型(Jones et al, 2016; Luo et al, 2015, 2017; Xia et al, 2013), 因
此地球系统模式中的陆地碳循环也存在本文描述 的四点内部特征与五类外部驱动因素。由于大部分 地球系统模式仍然不包含动态植被过程, 因此目前 仍难以研究陆地生态系统状态突变带来的碳循环 变化问题。但是, 如果未来的地球系统模型能准确 刻画植被群落动态(Franklin et al, 2020), 同时以 GEDI为代表的遥感卫星不断产生高精度的全球植 被结构数据(Qi \& Dubayah, 2016), 那么陆地碳循环 的动态非平衡状态将可能在较大的空间尺度上实 现其可预测性。

需要指出的是, 公式(1)是对生态系统水平碳 循环动态的一阶线性描述, 基于其变量可以引入一 系列非线性生态学过程。例如, 决定植物群落动态 的选择、漂移、扩散和物种形成等基本过程(Vellend, 2016)对生态系统的净初级生产力 $(U)$ 具有重要影 响。土壤微生物的非线性分解过程影响了土壤碳库 的分解速率, 从而使土壤碳库的分解过程在响应环 境变化时产生许多非线性现象。这些非线性响应现 象包括干旱生态系统土壤呼吸对降雨事件的脉冲 式响应(Liu et al, 2002)和新鲜碳输入对深层土壤有 机质分解的激发效应(Fontaine et al, 2003)等。以上 土壤有机碳分解的非线性过程可以被公式(1)中的 环境标量刻画。该环境标量具有较大的可扩展性, 可以包含温度、水分、养分与氧气等环境因子对土 壤有机质分解的非线性调控作用。因此，公式(1)可 以被进一步展开, 并结合特殊生态系统的生物群落 特征和环境特点, 构建其碳循环过程的数值模型。 
5 总结

陆地碳循环的动态非平衡状态假说为理解和 预测陆地碳汇动态提供了一个理论框架。基于该框 架, 我们认为全球陆地碳汇的未来变化动态遵循一 些基本原理。在年际尺度上, 单次干扰事件和极端 性天气/气候事件是陆地碳汇变化的重要外部驱动 力, 它们对陆地生态系统的碳汇功能影响主要在年 际或十年际的时间尺度上。在百年或更长的时间尺 度上, 干扰格局的改变是陆地碳循环的动态非平衡 的重要驱动因素。呈趋势性渐变的全球环境变化一 方面直接影响植物的光合作用与微生物的呼吸作 用, 从而影响生态系统碳汇动态; 另一方面间接地 改变生物多样性与群落结构, 进而重塑生态系统的 结构并对其碳汇功能产生影响。在干扰格局与生态 系统结构同时发生改变的情景下, 生态系统的状态 可能发生变迁, 从而使碳循环出现新的平衡态, 因 此使陆地碳循环的动态非平衡状态难以预测。因此, 我们认为未来的陆地碳循环研究需要开拓新的研 究方向, 以进一步探索碳循环内部特征与外部环境 驱动力之间的相互作用关系。这些方向包括单次干 扰事件对生产力、碳滞留时间与碳库动态的影响, 多重干扰事件的联合概率分布及其对碳循环关键 过程的影响, 生物群落结构变化与生态系统碳汇稳 定性的关系, 地球系统模式对干扰格局、群落结构 与碳循环过程耦合关系的模拟, 以及生态系统状态 突变与碳循环变迁的关系与机理等。

致谢: 感谢张大勇博士与王少鹏博士的约稿邀请和 崔二乾博士对参考文献的检校。

\section{参考文献}

Allison SD, Wallenstein MD, Bradford MA (2010) Soil-carbon response to warming dependent on microbial physiology. Nature Geoscience, 3, 336-340.

Baldocchi DD (2020) How eddy covariance flux measurements have contributed to our understanding of Global Change Biology. Global Change Biology, 26, 242-260.

Ballantyne A, Smith W, Anderegg W, Kauppi P, Sarmiento J, Tans P, Shevliakova E, Pan YD, Poulter B, Anav A, Friedlingstein P, Houghton R, Running S (2017) Accelerating net terrestrial carbon uptake during the warming hiatus due to reduced respiration. Nature Climate
Change, 7, 148-152.

Ballantyne AP, Alden CB, Miller JB, Tans PP, White JWC (2012) Increase in observed net carbon dioxide uptake by land and oceans during the past 50 years. Nature, 488, 70-72.

Bastos A, Ciais P, Friedlingstein P, Sitch S, Pongratz J, Fan L, Wigneron JP, Weber U, Reichstein M, Fu Z, Anthoni P, Arneth A, Haverd V, Jain AK, Joetzjer E, Knauer J, Lienert S, Loughran T, McGuire PC, Tian H, Viovy N, Zaehle S (2020) Direct and seasonal legacy effects of the 2018 heat wave and drought on European ecosystem productivity. Science Advances, 6, eaba2724.

Brando PM, Paolucci L, Ummenhofer CC, Ordway EM, Hartmann H, Cattau ME, Rattis L, Medjibe V, Coe MT, Balch J (2019) Droughts, wildfires, and forest carbon cycling: A pantropical synthesis. Annual Review of Earth and Planetary Sciences, 47, 555-581.

Burke IC, Kaye JP, Bird SP, Hall SA, McCulley RL, Sommerville GL (2003) Evaluating and testing models of terrestrial biogeochemistry: The role of temperature in controlling decomposition. In: Models in Ecosystem Science (Canham CD, Cole JJ, Lauenroth WK), pp. 225253. Princeton University Press, Princeton, New Jersey.

Cai A, Liang G, Zhang X, Zhang W, Li L, Rui Y, Xu M, Luo Y (2018) Long-term straw decomposition in agro-ecosystems described by a unified three-exponentiation equation with thermal time. Science of the Total Environment, 636, 699-708.

Chambers JQ, Fisher JI, Zeng H, Chapman EL, Baker DB, Hurtt GC (2007) Hurricane Katrina's carbon footprint on US Gulf Coast forests. Science, 318, 1107-1107.

Ciais P, Tan J, Wang X, Roedenbeck C, Chevallier F, Piao SL, Moriarty R, Broquet G, Le Quéré C, Canadell JG, Peng SH, Poulter B, Liu Z, Tans P (2019) Five decades of northern land carbon uptake revealed by the interhemispheric $\mathrm{CO}_{2}$ gradient. Nature, 568, 221-225.

Cox PM, Betts RA, Collins M, Harris PP, Huntingford C, Jones CD (2004) Amazonian forest dieback under climate-carbon cycle projections for the 21st century. Theoretical and Applied Climatology, 78, 137-156.

Cramer W, Bondeau A, Woodward FI, Prentice IC, Betts RA, Brovkin V, Cox PM, Fisher V, Foley JA, Friend AD, Kucharik C, Lomas MR, Ramankutty N, Sitch S, Smith B, White A, Young-Molling C (2001) Global response of terrestrial ecosystem structure and function to $\mathrm{CO}_{2}$ and climate change: Results from six dynamic global vegetation models. Global Change Biology, 7, 357-373.

Donohue I, Hillebrand H, Montoya JM, Petchey OL, Pimm SL, Fowler MS, Healy K, Jackson AL, Lurgi M, McClean D, O’Connor NE, O’Gorman EJ, Yang Q (2016) Navigating the complexity of ecological stability. Ecology Letters, 19, 1172-1185.

Du L, Mikle N, Zou ZH, Huang YY, Shi Z, Jiang LF, 
McCarthy HR, Liang JY, Luo YQ (2018) Global patterns of extreme drought-induced loss in land primary production: Identifying ecological extremes from rain-use efficiency. Science of the Total Environment, 628, 611-620.

Farquhar GD, Caemmerer S, Berry JA (1980) A biochemical model of photosynthetic $\mathrm{CO}_{2}$ assimilation in leaves of $\mathrm{C}_{3}$ species. Planta, 149, 78-90.

Fernández-Martínez M, Sardans J, Chevallier F, Ciais P, Obersteiner M, Vicca S, Canadell JG, Bastos A, Friedlingstein P, Sitch S, Piao SL, Janssens IA, Peñuelas J (2019) Global trends in carbon sinks and their relationships with $\mathrm{CO}_{2}$ and temperature. Nature Climate Change, 9, 73-79.

Fontaine S, Mariotti A, Abbadie L (2003) The priming effect of organic matter: A question of microbial competition? Soil Biology and Biochemistry, 35, 837-843.

Franklin O, Harrison SP, Dewar R, Farrior CE, Brännström Å, Dieckmann U, Pietsch S, Falster D, Cramer W, Loreau M, Wang H, Mäkelä A, Rebel KT, Meron E, Schymanski SJ, Rovenskaya E, Stocker BD, Zaehle S, Manzoni S, van Oijen M, Wright IJ, Ciais P, van Bodegom PM, Peñuelas J, Hofhansl F, Terrer C, Soudzilovskaia NA, Midgley G, Prentice IC (2020) Organizing principles for vegetation dynamics. Nature Plants, 6, 444-453.

Franks PJ, Adams MA, Amthor JS, Barbour MM, Berry JA, Ellsworth DS, Farquhar GD, Ghannoum O, Lloyd J, McDowell N, Norby RJ, Tissue DT, von Caemmerer S (2013) Sensitivity of plants to changing atmospheric $\mathrm{CO}_{2}$ concentration: From the geological past to the next century. New Phytologist, 197, 1077-1094.

Friedlingstein P, Jones MW, O’Sullivan M, Andrew RM, Hauck J, Peters GP, Peters W, Pongratz J, Sitch S, Le Quéré C, Bakker DCE, Canadell JG, Ciais P, Jackson RB, Anthoni P, Barbero L, Bastos A, Bastrikov V, Becker M, Bopp L, Buitenhuis E, Chandra N, Chevallier F, Chini LP, Currie KI, Feely RA, Gehlen M, Gilfillan D, Gkritzalis T, Goll DS, Gruber N, Gutekunst S, Harris I, Haverd V, Houghton RA, Hurtt G, Ilyina T, Jain AK, Joetzjer E, Kaplan JO, Kato E, Goldewijk KK, Korsbakken JI, Landschützer P, Lauvset S, Lefèvre N, Lenton A, Lienert S, Lombardozzi D, Marland G, McGuire PC, Melton JR, Metzl N, Munro DR, Nabel JEMS, Nakaoka S, Neill C, Omar AM, Ono T, Peregon A, Pierrot D, Poulter B, Rehder G, Resplandy L, Robertson E, Rödenbeck C, Séférian R, Schwinger J, Smith N, Tans PP, Tian HQ, Tilbrook B, Tubiello FN, Van der Werf GR, Wiltshire AJ, Zaehle S (2019) Global Carbon Budget 2019. Earth System Science Data, 11, 1783-1838.

Grace J, Mitchard E, Gloor E (2014) Perturbations in the carbon budget of the tropics. Global Change Biology, 20, 3238-3255.

Grosse G, Harden J, Turetsky M, McGuire AD, Camill P, Tarnocai C, Frolking S, Schuur EAG, Jorgenson T, Marchenko S, Romanovsky V, Wickland KP, French N,
Waldrop M, Bourgeau-Chavez L, Striegl RG (2011) Vulnerability of high-latitude soil organic carbon in north America to disturbance. Journal of Geophysical Research Biogeosciences, 116, G00K06.

Hastings A, Abbott KC, Cuddington K, Francis T, Gellner G, Lai Y-C, Morozov A, Petrovskii S, Scranton K, Zeeman ML (2018) Transient phenomena in ecology. Science, 361, 990.

Higgins SI, Scheiter S (2012) Atmospheric $\mathrm{CO}_{2}$ forces abrupt vegetation shifts locally, but not globally. Nature, 488, 209-212.

Holling CS (1959) Some characteristics of simple types of predation and parasitism. The Canadian Entomologist, 91, 385-398.

Holling CS (1973) Resilience and stability of ecological systems. Annual Review of Ecology and Systematics, 4, $1-23$.

Houghton RA, House JI, Pongratz J, van der Werf GR, DeFries RS, Hansen MC, Le Quéré C, Ramankutty N (2012) Carbon emissions from land use and land-cover change. Biogeosciences, 9, 5125-5142.

Hu FS, Higuera PE, Walsh JE, Chapman WL, Duffy PA, Brubaker LB, Chipman ML (2010) Tundra burning in Alaska: Linkages to climatic change and sea ice retreat. Journal of Geophysical Research: Biogeosciences, 115, G04002.

Hu MJ, Wan SQ (2019) Effects of fire and nitrogen addition on photosynthesis and growth of three dominant understory plant species in a temperate forest. Journal of Plant Ecology, 12, 759-768.

Huang K, Xia J (2019) High ecosystem stability of evergreen broadleaf forests under severe droughts. Global Change Biology, 25, 3494-3503.

Huang K, Xia JY, Wang YP, Ahlström A, Chen JQ, Cook RB, Cui EQ, Fang YY, Fisher JB, Huntzinger DN, Li Z, Michalak AM, Qiao Y, Schaefer K, Schwalm C, Wang J, Wei YX, Xu XN, Yan LM, Bian CY, Luo YQ (2018) Enhanced peak growth of global vegetation and its key mechanisms. Nature Ecology \& Evolution, 2, 1897-1905.

Humphrey V, Zscheischler J, Ciais P, Gudmundsson L, Sitch S, Seneviratne SI (2018) Sensitivity of atmospheric $\mathrm{CO}_{2}$ growth rate to observed changes in terrestrial water storage. Nature, 560, 628-631.

Isbell $\mathrm{F}$, Craven $\mathrm{D}$, Connolly $\mathrm{J}$, Loreau $\mathrm{M}$, Schmid $\mathrm{B}$, Beierkuhnlein C, Bezemer TM, Bonin C, Bruelheide H, de Luca E, Ebeling A, Griffin JN, Guo Q, Hautier Y, Hector A, Jentsch A, Kreyling J, Lanta V, Manning P, Meyer ST, Mori AS, Naeem S, Niklaus PA, Polley HW, Reich PB, Roscher C, Seabloom EW, Smith MD, Thakur MP, Tilman D, Tracy BF, van der Putten WH, van Ruijven J, Weigelt A, Weisser WW, Wilsey B, Eisenhauer N (2015) Biodiversity increases the resistance of ecosystem productivity to climate extremes. Nature, 526, 574-577.

Johnson CM, Zarin DJ, Johnson AH (2000) Post-disturbance 
aboveground biomass accumulation in global secondary forests. Ecology, 81, 1395-1401.

Jones CD, Arora V, Friedlingstein P, Bopp L, Brovkin V, Dunne J, Graven H, Hoffman F, Ilyina T, John JG, Jung M, Kawamiya M, Koven C, Pongratz J, Raddatz T, Randerson JT, Zaehle S (2016) C4MIP-The coupled climate-carbon cycle model intercomparison project: Experimental protocol for CMIP6. Geoscientific Model Development, 9, 2853-2880.

Jung M, Reichstein M, Schwalm CR, Huntingford C, Sitch S, Ahlström A, Arneth A, Camps-Valls G, Ciais P, Friedlingstein P, Gans F, Ichii K, Jain AK, Kato E, Papale D, Poulter B, Raduly B, Rödenbeck C, Tramontana G, Viovy N, Wang YP, Weber U, Zaehle S, Zeng N (2017) Compensatory water effects link yearly global land $\mathrm{CO}_{2}$ sink changes to temperature. Nature, 541, 516-520.

Jung M, Schwalm C, Migliavacca M, Walther S, Camps-Valls G, Koirala S, Anthoni P, Besnard S, Bodesheim P, Carvalhais N, Chevallier F, Gans F, Goll DS, Haverd V, Köhler P, Ichii K, Jain AK, Liu JZ, Lombardozzi D, Nabel JEMS, Nelson JA, O’Sullivan M, Pallandt M, Papale D, Peters W, Pongratz J, Rödenbeck C, Sitch S, Tramontana G, Walker A, Weber U, Reichstein M (2020) Scaling carbon fluxes from eddy covariance sites to globe: Synthesis and evaluation of the FLUXCOM approach. Biogeosciences, 17, 1343-1365.

Kelly R, Chipman ML, Higuera PE, Stefanova I, Brubaker LB, Hu FS (2013) Recent burning of boreal forests exceeds fire regime limits of the past 10,000 years. Proceedings of the National Academy of Sciences, USA, 110, 13055-13060.

Knapp AK, Avolio ML, Beier C, Carroll CJW, Collins SL, Dukes JS, Fraser LH, Griffin-Nolan RJ, Hoover DL, Jentsch A, Loik ME, Phillips RP, Post AK, Sala OE, Slette IJ, Yahdjian L, Smith MD (2017) Pushing precipitation to the extremes in distributed experiments: Recommendations for simulating wet and dry years. Global Change Biology, 23, 1774-1782.

Kurz WA, Dymond CC, Stinson G, Rampley GJ, Neilson ET, Carroll AL, Ebata T, Safranyik L (2008) Mountain pine beetle and forest carbon feedback to climate change. Nature, 452, 987-990.

Lader R, Walsh JE, Bhatt US, Bieniek PA (2017) Projections of twenty-first-century climate extremes for Alaska via dynamical downscaling and quantile mapping. Journal of Applied Meteorology \& Climatology, 56, 2393-2409.

Lawrence DM, Fisher RA, Koven CD, Oleson KW, Swenson SC, Bonan G, Collier N, Ghimire B, van Kampenhout L, Kennedy D, Kluzek E, Lawrence PJ, Li F, Li H, Lombardozzi D, Riley W, Sacks WJ, Shi M, Vertenstein M, Wieder WR, Xu C, Ali AA, Badger AM, Bisht G, van den Broeke M, Brunke MA, Burns SP, Buzen J, Clark M, Craig A, Dahlin K, Drewniak B, Fisher JB, Flanner M, Fox AM, Gentine P, Hoffman F, Keppel-Aleks G, Knox R, Kumar S,
Lenaerts J, Leung LR, Lipscomb WH, Lu Y, Pandey A, Pelletier JD, Perket J, Randerson JT, Ricciuto DM, Sanderson BM, Slater A, Subin ZM, Tang J, Thomas RQ, Martin MV, Zeng X (2020) The Community Land Model Version 5: Description of new features, benchmarking, and impact of forcing uncertainty. Journal of Advances in Modeling Earth Systems, 11, 4245-4287.

Liang J, Zhou Z, Huo C, Shi Z, Cole JR, Huang L, Konstantinidis KT, Li X, Liu B, Luo Z, Penton CR, Schuur EAG, Tiedje JM, Wang Y-P, Wu L, Xia J, Zhou J, Luo Y (2018) More replenishment than priming loss of soil organic carbon with additional carbon input. Nature Communications, 9, 3175.

Liu X, Wan S, Su B, Hui D, Luo Y (2002) Response of soil $\mathrm{CO}_{2}$ efflux to water manipulation in a tallgrass prairie ecosystem. Plant and Soil, 240, 213-223.

Luo YQ, Keenan TF, Smith M (2015) Predictability of the terrestrial carbon cycle. Global Change Biology, 21, 1737-1751.

Luo YQ, Shi Z, Lu XJ, Xia JY, Liang JY, Jiang J, Wang Y, Smith MJ, Jiang LF, Ahlström A, Chen B, Hararuk O, Hastings A, Hoffman F, Medlyn B, Niu SL, Rasmussen M, Todd-Brown K, Wang YP (2017) Transient dynamics of terrestrial carbon storage: Mathematical foundation and its applications. Biogeosciences, 14, 145-161.

Luo YQ, Weng ES (2011) Dynamic disequilibrium of the terrestrial carbon cycle under global change. Trends in Ecology \& Evolution, 26, 96-104.

Luo YQ, White LW, Canadell JG, DeLucia EH, Ellsworth DS, Finzi A, Lichter J, Schlesinger WH (2003) Sustainability of terrestrial carbon sequestration: A case study in Duke Forest with inversion approach. Global Biogeochemical Cycles, 17, 1021.

Lyapunov AM (1992) The general problem of the stability of motion. International Journal of Control, 55, 531-534.

Mack MC, Bret-Harte MS, Hollingsworth TN, Jandt RR, Schuur EAG, Shaver GR, Verbyla DL (2011) Carbon loss from an unprecedented Arctic tundra wilftre. Nature, 475, 489-492.

Mack MC, D’Antonio CM (1998) Impacts of biological invasions on disturbance regimes. Trends in Ecology \& Evolution, 13, 195-198.

Matamala R, Jastrow JD, Miller RM, Garten CT (2008) Temporal changes in $\mathrm{C}$ and $\mathrm{N}$ stocks of restored prairie: Implications for $\mathrm{C}$ sequestration strategies. Ecological Applications, 18, 1470-1488.

McDowell NG, Allen CD, Anderson-Teixeira K, Aukema BH, Bond-Lamberty B, Chini L, Slark JS, Dietze M, Grossiord C, Hanbury-Brown A, Hurtt GC, Jackson RB, Johnson DJ, Kueppers L, Lichstein JW, Ogle K, Poulter B, Pugh TAM, Seidl R, Turner MG, Uriarte M, Walker AP, Xu C (2020) Pervasive shifts in forest dynamics in a changing world. Science, 368, eaaz9463. 
Melillo JM, Frey SD, DeAngelis KM, Werner WJ, Bernard MJ, Bowles FP, Pold G, Knorr MA, Grandy AS (2017) Long-term pattern and magnitude of soil carbon feedback to the climate system in a warming world. Science, 358, 101-105.

Morozov A, Abbott K, Cuddington K, Francis T, Gellner G, Hastings A, Lai Y-C, Petrovskii S, Scranton K, Zeeman ML (2020) Long transients in ecology: Theory and applications. Physics of Life Reviews, 32, 1-40.

Odum EP (1969) The strategy of ecosystem development. Science, 164, 262-270.

Parton WJ, Schimel DS, Cole CV, Ojima DS (1987) Analysis of factors controlling soil organic matter levels in Great Plains grasslands. Soil Science Society of America Journal, 51, 1173-1179.

Piao SL, Wang XH, Park T, Chen C, Lian X, He Y, Bjerke JW, Chen AP, Ciais P, Tømmervik H, Nemani RR, Myneni RB (2020) Characteristics, drivers and feedbacks of global greening. Nature Reviews Earth \& Environment, 1, 14-27.

Pires APF, Srivastava DS, Farjalla VF (2018) Is biodiversity able to buffer ecosystems from climate change? What we know and what we don't. BioScience, 68, 273-280.

Qi WL, Dubayah RO (2016) Combining Tandem-X InSAR and simulated GEDI lidar observations for forest structure mapping. Remote Sensing of Environment, 187, 253-266.

Rasmussen M, Hastings A, Smith MJ, Agusto FB, Chen-Charpentier BM, Hoffman FM, Jiang J, Todd-Brown KEO, Wang Y, Wang Y-P, Luo Y (2016) Transit times and mean ages for nonautonomous and autonomous compartmental systems. Journal of Mathematical Biology, 73, 1379-1398.

Reichstein M, Bahn M, Ciais P, Frank D, Mahecha MD, Seneviratne SI, Zscheischler J, Beer C, Buchmann N, Frank DC, Papale D, Rammig A, Smith P, Thonicke K, van der Velde M, Vicca S, Walz A, Wattenbach M (2013) Climate extremes and the carbon cycle. Nature, 500, 287-295.

Resco de Dios V (2020) Forest succession, alternative states, and fire-vegetation feedbacks. In: Plant-Fire Interactions: Managing Forest Ecosystems, Vol. 36 (eds von Gadow K, Pukkala T, Tomé M). Springer, Cham.

Rödenbeck C, Zaehle S, Keeling R, Heimann M (2018) How does the terrestrial carbon exchange respond to inter-annual climatic variations? A quantification based on atmospheric $\mathrm{CO}_{2}$ data. Biogeosciences, 15, 2481-2498.

Running SW (2008) Ecosystem disturbance, carbon, and climate. Science, 321, 652-653.

Scheffer M, Carpenter S, Foley JA, Folke C, Walker B (2001) Catastrophic shifts in ecosystems. Nature, 413, 591-596.

Schuur EAG, McGuire AD, Schädel C, Grosse G, Harden JW, Hayes DJ, Hugelius G, Koven CD, Kuhry P, Lawrence DM, Natali SM, Olefeldt D, Romanovsky VE, Schaefer K, Turetsky MR, Treat CC, Vonk JE (2015) Climate change and the permafrost carbon feedback. Nature, 520, 171-179.
Schuur EAG, Vogel JG, Crummer KG, Lee H, Sickman JO, Osterkamp TE (2009) The effect of permafrost thaw on old carbon release and net carbon exchange from tundra. Nature, 459, 556-559.

Silver WL, Miya RK (2001) Global patterns in root decomposition: Comparisons of climate and litter quality effects. Oecologia, 129, 407-419.

Shi Z, Allison SD, He Y, Levine PA, Hoyt AM, BeeM-Miller J, Zhu Q, Wieder WR, Trumbore S, Randerson JT (2020) The age distribution of global soil carbon inferred from radiocarbon measurements. Nature Geoscience, 13, 555-559.

Song J, Wan SQ, Piao SL, Knapp AK, Classen AT, Vicca S, Ciais P, Hovenden MJ, Leuzinger S, Beier C, Kardol P, Xia JY, Liu Q, Ru JY, Zhou ZX, Luo YQ, Guo DL, Langley JA, Zscheischler J, Dukes JS, Tang JW, Chen JQ, Hofmockel KS, Kueppers LM, Rustad L, Liu LL, Smith MD, Templer PH, Thomas RQ, Norby RJ, Phillips RP, Niu SL, Fatichi S, Wang YP, Shao PS, Han HY, Wang DD, Lei LJ, Wang J, Li XN, Zhang Q, Li XM, Su FL, Liu B, Yang F, Ma G, Li GY, Liu YC, Liu YZ, Yang ZL, Zhang KS, Miao Y, Hu MJ, Yan C, Zhang A, Zhong MX, Hui Y, Li Y, Zheng MM (2019) A meta-analysis of 1,119 manipulative experiments on terrestrial carbon-cycling responses to global change. Nature Ecology \& Evolution, 3, 1309-1320.

Staver AC, Archibald S, Levin SA (2011) The global extent and determinants of savanna and forest as alternative biome states. Science, 334, 230-232.

Staver AC, Levin SA (2012) Integrating theoretical climate and fire effects on savanna and forest systems. The American Naturalist, 180, 211-224.

Tagesson T, Schurgers G, Horion S, Ciais P, Tian F, Brandt M, Ahlström A, Wigneron J-P, Ardö J, Olin S, Fan L, Wu Z, Fensholt R (2020) Recent divergence in the contributions of tropical and boreal forests to the terrestrial carbon sink. Nature Ecology \& Evolution, 4, 202-209.

Torn MS, Trumbore SE, Chadwick OA, Vitousek PM, Hendricks DM (1997) Mineral control of soil organic carbon storage and turnover. Nature, 389, 170-173.

Trumbore S (2009) Radiocarbon and soil carbon dynamics. Annual Review of Earth and Planetary Sciences, 37, 47-66.

Turetsky MR, Abbott BW, Jones MC, Anthony KW, Olefeldt D, Schuur EAG, Grosse G, Kuhry P, Hugelius G, Koven C, Lawrence DM, Gibson C, Sannel ABK, McGuire AD (2020) Carbon release through abrupt permafrost thaw. Nature Geoscience, 13, 138-143.

Vanderwel MC, Coomes DA, Purves DW (2013) Quantifying variation in forest disturbance, and its effects on aboveground biomass dynamics, across the eastern United States. Global Change Biology, 19, 1504-1517.

Vellend M (2016) The Theory of Ecological Communities. Princeton University Press, Princeton.

Walker XJ, Baltzer JL, Cumming SG, Day NJ, Ebert C, Goetz 
S, Johnstone JF, Potter S, Rogers BM, Schuur EAG, Turetsky MR, Mack MC (2019) Increasing wildfires threaten historic carbon sink of boreal forest soils. Nature, 572, 520-523.

Wang YP, Chen BC, Wieder WR, Leite M, Medlyn BE, Rasmussen M, Smith MJ, Agusto FB, Hoffman F, Luo YQ (2014) Oscillatory behavior of two nonlinear microbial models of soil carbon decomposition. Biogeosciences, 11, 1817-1831.

Weintraub MN, Schimel JP (2003) Interactions between carbon and nitrogen mineralization and soil organic matter chemistry in arctic tundra soils. Ecosystems, 6, 129-143.

Weng ES, Luo YQ, Wang WL, Wang H, Hayes DJ, McGuire AD, Hastings A, Schimel DS (2012) Ecosystem carbon storage capacity as affected by disturbance regimes: A general theoretical model. Journal of Geophysical Research: Biogeosciences, 117, G03014.

Wieder WR, Bonan GB, Allison SD (2013) Global soil carbon projections are improved by modelling microbial processes. Nature Climate Change, 3, 909-912.

Williams CA, Collatz GJ, Masek J, Goward SN (2012) Carbon consequences of forest disturbance and recovery across the conterminous United States. Global Biogeochemical Cycles, 26, GB1005.

Xia J, Luo Y, Wang YP, Hararuk O (2013) Traceable components of terrestrial carbon storage capacity in biogeochemical models. Global Change Biology, 19, 2104-2116.

Xia J, Wang J, Niu S (2020) Research challenges and opportunities for using big data in global change biology. Global Change Biology, 26, 6040-6061.
Xu X, Shi Z, Li D, Ray A, Ruan H, Craine JM, Liang J, Zhou J, Luo Y (2016) Soil properties control decomposition of soil organic carbon: Results from data-assimilation analysis. Geoderma, 262, 235-242.

Yang YH, Luo YQ, Finzi AC (2011) Carbon and nitrogen dynamics during forest stand development: A global synthesis. New Phytologist, 190, 977-989.

Yu K, Smith WK, Trugman AT, Condit R, Hubbell SP, Sardans J, Peng CH, Zhu K, Peñuelas J, Cailleret M, Levanic T, Gessler A, Schaub M, Ferretti M, Anderegg WRL (2019) Pervasive decreases in living vegetation carbon turnover time across forest climate zones. Proceedings of the National Academy of Sciences, USA, 116, 24662-24667.

Zhang DQ, Hui DF, Luo YQ, Zhou GY (2008) Rates of litter decomposition in terrestrial ecosystems: Global patterns and controlling factors. Journal of Plant Ecology, 1, 85-93.

Zhu K, Zhang J, Niu S, Chu C, Luo Y (2018) Limits to growth of forest biomass carbon sink under climate change. Nature Communications, 9, 2709.

Zhu ZC, Piao SL, Myneni RB, Huang MT, Zeng ZZ, Canadell JC, Ciais P, Sitch S, Friedlingstein P, Arneth A, Cao CX, Cheng L, Kato E, Koven C, Li Y, Lian X, Liu YW, Liu RG, Mao JF, Pan YZ, Peng SS, Peñuelas J, Poulter B, Pugh TAM, Stocker BD, Viovy N, Wang XH, Wang YP, Xiao ZQ, Yang H, Zaehle S, Zeng N (2016) Greening of the Earth and its drivers. Nature Climate Change, 6, 791-795.

(责任编委: 王少鹏 责任编辑: 周玉荣) 